\title{
Transseptal Left Heart Catheterization as an Aid in the Diagnosis of Cor Triatriatum
}

\author{
Elizabeth M. Shaffer, Albert P. Rocchini, Macdonald Dick II, and Amnon Rosenthal \\ Department of Pediatric Cardiology, University of Michigan Medical Center, Ann Arbor, Michigan, USA \\ SUMMARY. A case of cor triatriatum is described in which transseptal left heart catheteriza- \\ tion was useful in making the diagnosis by allowing easy access to the accessory chamber for \\ pressure measurements and angiography.
}

\section{KEY WORDS: Transseptal catheterization - Cor triatriatum}

Cor triatriatum is an unusual form of congenital heart disease that frequently causes pulmonary venous obstruction [4]. Diagnosis is made at cardiac catheterization by the presence of an elevated pulmonary artery pressure and a gradient between the pulmonary capillary wedge pressure (PCWP) and the left ventricular end diastolic pressure (LVEDP). The main differential diagnoses are mitral stenosis, supravalvar mitral ring, and obstructed pulmonary veins (single or multiple). A pulmonary arterial angiogram will sometimes define the left atrial membrane but frequently it is not identified $[3,5]$.

The technique of transseptal left heart catheterization has been used in clinical cardiology since $1959[1,8]$. Modifications to the original technique have been described and its usefulness and safety have been reported for infants and children with congenital heart disease [6]. The technique has been reported primarily for patients with left ventricular outflow tract obstruction, but it has proven useful in a variety of other cardiac defects $[2,6]$.

We describe a case of cor triatriatum in which transseptal left heart catheterization was useful in making the diagnosis by allowing easy access to the accessory chamber for pressure measurements and angiography.

\footnotetext{
Address reprint requests to: Dr. Elizabeth M. Shaffer. Pediatric Cardiology, University of Michigan Medical Center, Room F1609, Box 0204, 1500 East Medical Center Drive, Ann Arbor, MI 48109, USA.
}

\section{Case Report}

M.E. is a 2-year-old child who was well until 1 month prior to admission when she developed symptoms suggestive of an upper respiratory tract infection. She was evaluated by her pediatrician, who heard a murmur for the first time. She was treated with antibiotics with resolution of her symptoms. When seen in follow-up, the murmur was still present and she was referred to C.S. Mott Children's Hospital for further evaluation. Past medical history revealed few upper respiratory tract infections and no history of pneumonia or wheezing. Physical examination revealed a healthy-appearing black female without cyanosis. Her weight was $10.4 \mathrm{~kg}(<5 \%)$ with a height of $84 \mathrm{~cm}(5 \%)$. She was mildly tachypneic (respiratory rate 28 breaths per minute), but had no rales or wheezes. Cardiac examination revealed a left precordial prominence, hyperdynamic apical and xiphoid impulses, a normal first heart sound, and a loud single second heart sound with a constant systolic ejection click at the left upper sternal border. A grade $3 / 6$ systolic, blowing, regurgitant murmur was heard best at the lower left sternal border and the apex. No diastolic murmurs were heard. The electrocardiogram revealed right axis deviation $(+120)$, left atrial enlargement, and biventricular hypertrophy (right greater than left). The chest radiograph showed moderate cardiac enlargement with pulmonary edema. The two-dimensional echocardiogram revealed cor triatriatum. The membrane attached to the atrial septum in the area of the fossa ovalis and the lateral wall of the left atrium. The pulmonary veins drained to the accessory chamber (Fig. 1). A cardiac catheterization was done that revealed pulmonary artery hypertension (70/30 $\mathrm{mmHg}$, mean $46 \mathrm{mmHg}$ ), elevated PCWP (a wave 26 $\mathrm{mmHg}$, mean $26 \mathrm{mmHg}$ ), and a normal LVEDP (12 $\mathrm{mmHg}$ ). The pressure in the accessory chamber was similar to the PCWP with a mean of $25 \mathrm{mmHg}$. The true left atrium was not entered. There were no natural openings between the right atrium and the true left atrium nor any communication between the right atrium and the accessory chamber. The transseptal approach was used to gain access to the high pressure accessory chamber and pulmonary veins. Cineangiography was done in the accessory chamber to define the intraatrial membrane (Fig. 2). 


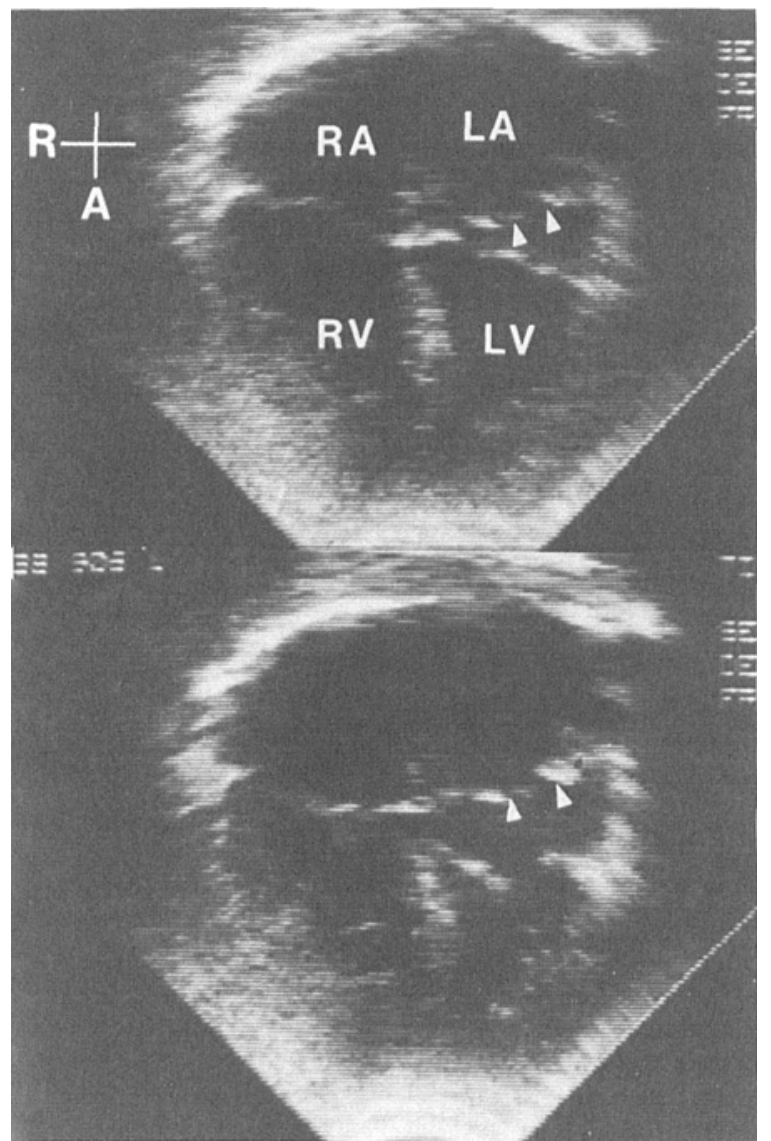

Fig. 1. Echocardiogram of the apical four-chamber view depicting the left atrial membrane (arrows) in systole (top frame) and diastole (bottom frame): $R$, right; $A$, apex; $R A$, right atrium; $L A$, left atrium; $R V$, right ventricle; and $L V$ left ventricle.

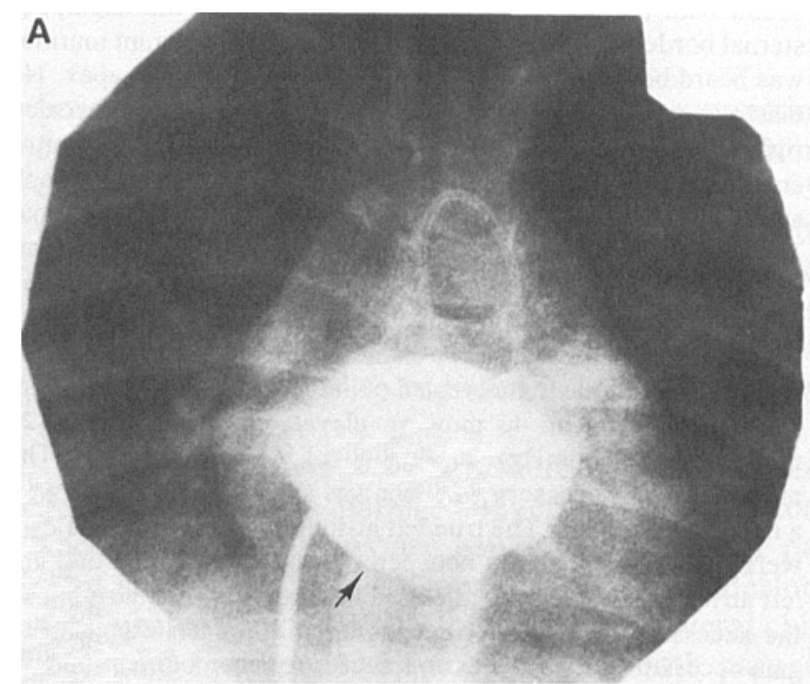

Fig. 2. The anterior posterior (A) and lateral (B) angiograms with the catheter positioned in the accessory chamber through the transseptal puncture demonstrates the intraatrial membrane (arrow).

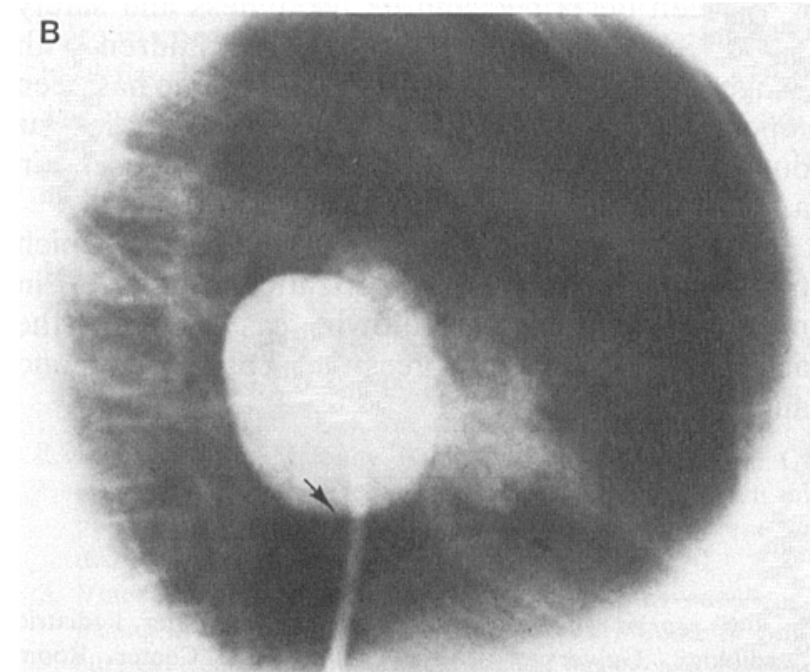

The patient was taken to the operating room 2 days after cardiac catheterization. On cardiopulmonary bypass the left atrial membrane was resected. Her recovery was uneventful. She is asymptomatic with no evidence of any cardiovascular abnormality 15 months after surgery.

\section{Discussion}

Cor triatriatum is an unusual form of congenital heart disease that usually presents in the first few years of life with dyspnea and multiple respiratory infections. Frequently the patients are considered to have primary lung disease.

In the classic form of cor triatriatum, the pulmonary veins enter an accessory chamber that joins the left atrium through a narrow opening. There are, however, multiple variations of cor triatriatum, including accessory chambers receiving all of the the pulmonary veins and not communicating with the left atrium and subtotal cor triatriatum where an accessory chamber receives part of the pulmonary veins and connects to the left atrium [4].

Diagnosis can be difficult. Two-dimensional echocardiography has enhanced our diagnostic capabilities with visualization of the membrane in the left atrium [9]. Cardiac catheterization and angiography remain the standards for diagnosis, but even these may be less than ideal. Pulmonary artery hypertension with elevated pulmonary capillary wedge pressures is suggestive of left ventricular inflow obstruction but not diagnostic of cor triatriatum. There may be a communication between the right atrium and the accessory chamber [7] in which case the PCWP may be normal. Pulmonary 
arterial angiograms may reveal the intraatrial diaphragm, but more frequently are disappointing. Transseptal left heart catheterization may aid in the diagnosis of cor triatriatum by allowing direct access to the accessory chamber for pressure measurements and angiography. The transseptal catheter is most easily directed posteriorly, which increases the probability of entering the accessory chamber. In our experience, entrance into the accessory chamber was easily obtained, but the true left atrium could not be entered from the accessory chamber. This may relate to the orientation of the opening or openings between the accessory chamber and the left atrium. If a catheter could be positioned in the left atrium through the accessory chamber, balloon dilation of a stenotic orifice between the chambers may be possible and allow decompression of the pulmonary veins.

In summary, we present a case of cor triatriatum in which transseptal left heart catheterization aided in the diagnosis by allowing access to the accessory chamber for pressure measurements and angiography. We recommend the use of this technique in patients with possible cor triatriatum.

\section{References}

1. Cope C (1959) Technique for transseptal catheterization of the left atrium: preliminary report. I Thorac Surg 37:482-486

2. Duff DF, Mullins CE (1978) Transseptal left heart catheterization in infants and children. Cath Cardiovasc Diagn 4:213223

3. Jacobstein MD, Hirschfeld SS (1982) Concealed left atrial membrane: pitfalls in the diagnosis of cor triatriatum and supravalve mitral ring. Am J Cardiol 49:780-786

4. Lucas RV (1983) Anomalous venous connections, pulmonary and systemic. In: Adams FH, Emmanouilides GC (eds) Moss heart disease in infants, children, and adolescents 1983. Williams and Wilkins, Baltimore, pp 475-479

5. Marin-Garcia J, Tandon R, Lucas RV, Edwards JE (1975) Cor triatriatum: study of 20 cases. Am J Cardiol 35:59-66

6. Mullins CE (1983) Transseptal left heart catheterization: experience with a new technique in 520 pediatric and adult patients. Pediatr Cardiol 4:239-246

7. Niwayama G (1960) Review cor triatriatum. Am Heart $J$ $59: 291-317$

8. Ross J Jr, Braunwald E, Morrow AG (1959) Transseptal left atrial puncture: new technique for the measurement of left atrial pressure in man. Am J Cardiol 3:653-655

9. Snider AR, Roge CL, Schiller NB, Silverman NH (1980) Congenital left ventricular inflow obstruction evaluated by twodimensional echocardiography. Circulation 61:848-855 